\title{
A NOTE ON LIMITS OF UNITARILY EQUIVALENT OPERATORS \\ BY
}

\author{
LAWRENCE A. FIALKOW
}

In loving memory of my mother

\begin{abstract}
Let $U(\mathcal{I C})$ denote the set of all unitary operators on a separable complex Hilbert space $\mathcal{H}$. If $T$ is a bounded linear operator on $\mathcal{K}$, let $\pi_{T}$ denote the mapping of $U(\mathcal{H C})$ onto $U(T)$ given by conjugation. It is proved that if $T$ is normal or isometric, then there exists a locally defined continuous cross-section for $\pi_{T}$ if and only if the spectrum of $T$ is finite. Examples of nonnormal operators with local cross-sections are given.
\end{abstract}

1. Introduction. Let $\mathcal{H}$ denote a separable complex Hilbert space and let $\mathcal{L}(\mathcal{H C})$ denote the algebra of all bounded linear operators on $\mathcal{H}$. Let $\mathcal{Q}(\mathcal{H C})$ denote the set of all unitary operators on $\mathcal{H C}$, and for $T$ in $\mathcal{L}(\mathcal{H C})$, let $Q(T)$ denote the unitary orbit of $T$, i.e., $\mathcal{Q}(T)=\left\{U^{*} T U \mid U\right.$ in $\left.\mathcal{Q}(\mathcal{H})\right\}$. We denote by $\pi_{T}$ the norm continuous mapping of $U(\mathcal{H C})$ onto $U(T)$ defined by $\pi_{T}(U)=U^{*} T U(U$ in $U(\mathscr{H}))$. A local cross-section for $\pi_{T}$ is a pair $\left(\varphi_{T}, \mathscr{B}\right)$

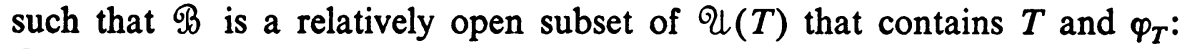
$\mathscr{B} \rightarrow \mathcal{Q}(\mathcal{H C})$ is a norm continuous function such that $\varphi_{T}(T)=1$ and $\pi_{T}\left(\varphi_{T}(S)\right)=S$ for each $S$ in $\mathscr{B}$. If $\pi_{T}$ has a local cross-section, we say that $T$ has a (local unitary) cross-section, and in this case $T$ clearly satisfies the following sequential unitary lifting property:

(P) If $\left\{U_{n}\right\} \subset \mathcal{Q}(\mathcal{F C})$ and $\lim \left\|U_{n}^{*} T U_{n}-T\right\|=0$, then there exists a sequence $\left\{W_{n}\right\} \subset \mathcal{Q}(\mathcal{H})$ such that $\lim \left\|W_{n}-1\right\|=0$ and such that $W_{n}^{*} T W_{n}$ $=U_{n}^{*} T U_{n}$ for each $n$. The problem to which this note is addressed is that of obtaining necessary and sufficient conditions for an operator on $\mathcal{H C}$ to have a local cross-section or to satisfy (P).

The fact that an orthogonal projection satisfies $(\mathrm{P})$ has appeared in the literature in diverse contexts. In [4, Lemma 1], P. R. Halmos proved that if $P$ and $Q$ are finite rank projections and $\|P-Q\|=\varepsilon<1$, then there exists a unitary operator $W$ such that $W^{*} P W=Q$ and $\|W-1\|<2 \varepsilon^{1 / 2}$. This result was used in proving a principal characterization of quasi-triangular operators [4, Theorem 2]. Earlier results related to this lemma appeared in [1]. As we remark below, this lemma implies that a finite rank projection satisfies (P).

Received by the editors April 24, 1975 and, in revised form, February 13, 1976.

AMS (MOS) subject classifications (1970). Primary 47A10.

- American Mathematical Society 1977 
An extension of the lemma to arbitrary projections in $\mathcal{L}(\mathcal{C})$ was given in [2] as a tool in studying the closure of the unitary orbit of a partial isometry.

In $\$ 3$ we prove that if an operator $T$ is normal or isometric, then it satisfies (P) if and only if its spectrum is finite; if the spectrum of $T$ is finite, then there exists a local cross-section $\left(\varphi_{T}, \mathscr{B}\right)$ such that for each operator $S$ in $\mathscr{B}$, $\varphi_{T}(S)$ is in the norm closed subalgebra of $\mathcal{L}(\mathcal{H})$ generated by $S, T$, and 1 . Furthermore, if a normal operator with infinite spectrum is a direct summand of an operator $S$, then $S$ does not satisfy (P). We prove in Theorem 3.5 that if $T$ is an irreducible operator and $T$ has a reducing essential eigenvalue, then $T$ does not satisfy (P); if such an operator is a direct summand of an operator $S$, then $S$ does not satisfy (P). This result is used to show that there exist compact, quasi-nilpotent operators that do not satisfy $(\mathrm{P})$; these include the Volterra integral operator and every compact injective bilateral weighted shift.

In $\$ \S 2$ and 4 we show that there do exist nonnormal operators that satisfy (P) or have local unitary cross-sections. In Corollary 2.4 we prove that each operator on a finite dimensional Hilbert space satisfies (P). Suppose that $T$ has a cross-section and that $S$ is an operator such that $T^{*} T+S^{*} S$ is invertible. In Theorems 4.2 and 4.3 we prove that the operator on $\mathcal{H} \oplus \mathcal{H}$ whose operator matrix is $\left(\begin{array}{c}T \\ S\end{array} 0\right)$ has a cross-section if either (1) $S$ is a right invertible operator in the $C^{*}$-algebra generated by $T$, or (2) $S$ is an isometry. Additional examples of operators with cross-sections may be constructed by application of Theorem 2.8, which states that a finite direct sum of operators (having mutually disjoint spectra) has a cross-section if and only if each summand has a cross-section. Analogues of these results for the sequential unitary lifting property are also given. In [2] it was proven that if $T$ has a local cross-section, then each path in $\mathcal{Q}(T)$ can be lifted to a path in $\mathcal{U}(\mathcal{H})$; we prove in Proposition 2.2 that if $T$ satisfies $(\mathrm{P})$, then $\mathscr{Q}(T)^{-}$(the norm closure of $\mathscr{U}(T)$ in $\mathcal{L}(\mathcal{H})$ ) is path connected (cf. [3]). At the end of $\S 4$ we list some questions related to characterizing the operators having local cross-sections.

I wish to thank E. Azoff, L. Brown, D. Hadwin, C. Pearcy, and the referee for their helpful comments and suggestions concerning this paper.

2. On the sequential unitary lifting property. In this section we give some general properties of operators satisfying $(\mathrm{P})$. We denote the spectrum of an operator $T$ by $\sigma(T)$. For $\varepsilon>0$, we denote the set $\{X$ in $U(T) \mid\|X-T\|<$ $\varepsilon\}$ by $\Re(T, \varepsilon)$.

LEMMA 2.1. If $U$ is a unitary operator in $\mathcal{E}(\mathcal{H})$ and $\|U-1\|<2$, then there exists a path $\left\{U_{t}\right\}_{0<t<1} \subset \mathcal{U}(\mathcal{H})$ such that $U_{0}=U, U_{1}=1$, and such that, for each $t,\left\|U_{t}-1\right\|<\|U-1\|$. 
Proof. Let $A$ denote a selfadjoint operator such that $U=e^{i A}$ (see [5, p. 66]). Since $2>\|U-1\|=\left\|e^{i A}-1\right\|=\sup _{s \in \sigma(A)}\left|e^{i s}-1\right|$, it is clear that $\sigma(A)$ contains no number of the form $\pi+2 \pi n$, where $n$ is an integer. For each $n$, let $I_{n}=[2 \pi n, 2 \pi n+\pi]$ and $J_{n}=[2 \pi n+\pi, 2 \pi(n+1)]$. For $0<t<$ 1 and $s$ in $I_{n} \cap \sigma(A)$, we define $f_{t}(s)=(1-t) s+2 \pi n t$; for $s$ in $J_{n} \cap \sigma(A)$, we define $f_{t}(s)=(1-t) s+2 \pi(n+1) t$. For $0<t<1$ and $s$ in $\sigma(A)$, we set $g_{t}(s)=e^{i f_{t}(s)}$. The hypothesis implies that $g_{t}$ is well defined and continuous on $\sigma(A)$, and it follows readily from the functional calculus that $U_{t}=g_{t}(A)$ $(0 \leqslant t \leqslant 1)$ defines a path satisfying the desired properties.

If $T$ is in $\mathcal{L}(\mathcal{F C})$, let $\delta(T)$ denote the set of all sequences $\left\{U_{n}\right\} \subset \mathcal{U}(\mathcal{F C})$ such that $\lim \left\|U_{n}^{*} T U_{n}-T\right\|=0$. If $U=\left\{U_{n}\right\}$ is in $\delta(T)$ and $V=\left\{V_{n}\right\} \subset$ Q (FC), we say that $V$ re-implements $U$ in case $\lim \left\|1-V_{n}\right\|=0$ and $U_{n}^{*} T U_{n}$ $=V_{n}^{*} T V_{n}$ for each $n$.

Proposition 2.2. If an operator $T$ in $\mathcal{L}(\mathcal{H})$ satisfies $(\mathrm{P})$, then $U(T)^{-}$is path connected.

Proof. Let $S$ be in $थ(T)^{-}$and let $\left\{U_{n}\right\} \subset \mathcal{Q}(\mathcal{F})$ be a sequence such that $\lim \left\|U_{n} T U_{n}^{*}-S\right\|=0$. Then $U=\left\{U_{n}^{*} U_{n+1}\right\}$ is in $\delta(T)$ and the hypothesis implies that there is a sequence $\left\{W_{n}\right\} \subset \mathcal{U}(\mathcal{H C})$ such that $\left\{W_{n}^{*}\right\}$ re-implements $U$. If $T_{n}=U_{n} T U_{n}^{*}$ and $V_{n}=U_{n+1} W_{n}^{*} U_{n+1}^{*}$, then $T_{n+1}=V_{n} T_{n} V_{n}^{*}$ and $\lim \left\|1-V_{n}\right\|=\lim \left\|1-W_{n}\right\|=0$. We may thus assume that $\left\|1-V_{n}\right\|<2$, and the preceding lemma implies that, for fixed $n$, there exists a path $\left\{V_{t, n}\right\}$ $(0<t<1)$ from $V_{n}$ to 1 such that $\left\|V_{t, n}-1\right\|<\left\|V_{n}-1\right\|$ for each $t$. It follows that $K_{n}=\left\{V_{t, n} T_{n} V_{t, n}^{*}\right\}(0 \leqslant t \leqslant 1)$ is a path from $T_{n+1}$ to $T_{n}$ such that for each $t$ we have

$$
\begin{aligned}
\left\|V_{t, n} T_{n} V_{t, n}^{*}-T_{n}\right\| & \leqslant\left\|V_{t, n} T_{n} V_{t, n}^{*}-V_{t, n} T_{n}\right\|+\left\|V_{t, n} T_{n}-T_{n}\right\| \\
& \leqslant\left\|V_{t, n} T_{n}\right\|\left\|V_{t, n}^{*}-1\right\|+\left\|V_{t, n}-1\right\|\left\|T_{n}\right\| \\
& \leqslant 2\|T\|\left\|V_{t, n}-1\right\| \leqslant 2\|T\|\left\|V_{n}-1\right\| .
\end{aligned}
$$

Thus $\left\|V_{t, n} T_{n} V_{t, n}^{*}-S\right\| \leqslant 2\|T\|\left\|V_{n}-1\right\|+\left\|T_{n}-S\right\|$, and it follows that the paths $\left\{K_{n}\right\}$ may be combined and reparametrized to form a single path from $T$ to $S$, each member of which, except perhaps $S$ itself, is in $U(T)$.

LEMMA 2.3. For an operator $T$ in $\mathcal{L}(\mathcal{H})$ the following properties are equivalent:

(P1) Given $\varepsilon>0$, there exists $\delta>0$ such that if $S$ is in $\mathcal{Q}(T)$ and $\|S-T\|<\delta$, then there exists a unitary operator $W$ such that $\|W-1\|<\varepsilon$ and $W^{*} T W=S$.

(P2) $T$ satisfies property $(\mathrm{P})$.

(P3) If $\left\{U_{n}\right\}$ is in $\delta(T)$, then there exists a subsequence $V=\left\{U_{n_{k}}\right\}$ and a sequence $W=\left\{W_{k}\right\}$ in $U(\mathcal{H})$ such that $W$ re-implements $V$. 
Proof. To prove that (P1) implies (P2), suppose that $U=\left\{U_{n}\right\}$ is in $\delta(T)$. For each integer $k>0$, corresponding to $\varepsilon=1 / k$, there exists $\delta_{k}>0$ satisfying the conditions of (P1). Let $N_{k}$ be a positive integer such that if $n>N_{k}$, then $\left\|U_{n}^{*} T U_{n}-T\right\|<\delta_{k}$; we may also assume that $N_{k}>N_{k-1}$ for each $k$. Thus, for $n \geqslant N_{k}$, there exists a unitary operator $W_{n, k}$ such that $\left\|W_{n, k}-1\right\|$ $<1 / k$ and $W_{n, k}^{*} T W_{n, k}=U_{n}^{*} T U_{n}$. For each integer $n \geqslant N_{1}$, let $k(n)$ denote the unique integer such that $N_{k(n)+1}>n>N_{k(n)}$, and define $W_{n}=W_{n, k(n)}$; for each $n<N_{1}$, let $W_{n}=U_{n}$. It now follows readily that $\left\{W_{n}\right\}$ re-implements $U$, and therefore $T$ satisfies (P).

It is clear that (P2) implies (P3). That (P3) implies (P1) may be proven by a straightforward proof by contradiction; we omit the details.

REMARK. To prove that an operator $T$ satisfies (P1), it clearly suffices to assume that $0<\varepsilon<2$. If $T$ is a finite rank projection and $0<\varepsilon<2$, the above-mentioned result of P. R. Halmos shows that $T$ satisfies (P1) with $\delta=\varepsilon^{2} / 4$.

Corollary 2.4. If $\mathcal{H}$ is a finite dimensional Hilbert space and $T$ is in $\mathcal{L}(\mathcal{H})$, then $T$ satisfies $(\mathrm{P})$.

Proof. Let $U=\left\{U_{n}\right\}$ be in $\delta(T)$. Since $U(\mathcal{H C})$ is compact, there exists a norm-convergent subsequence $U_{n_{k}} \rightarrow V$. Now $V$ is a unitary operator that commutes with $T$, and it follows that $\left\{V^{*} U_{n_{k}}\right\}$ re-implements $\left\{U_{n_{k}}\right\}$. Thus $T$ satisfies (P3), and Lemma 2.3 implies that $T$ satisfies (P).

Let $\mathcal{K}_{1}$ and $\mathcal{F}_{2}$ denote separable complex Hilbert spaces and let $\mathcal{L}\left(\mathcal{F}_{2}, \mathcal{H}_{1}\right)$ denote the Banach space of all bounded linear operators from $\mathcal{F}_{2}$ into $\mathcal{K}_{1}$. For operators $A$ in $\mathcal{L}\left(\mathcal{K}_{1}\right)$ and $B$ in $\mathcal{L}\left(\mathcal{H}_{2}\right)$ we define the operator $\mathcal{T}$ on $\mathcal{L}\left(\mathcal{F}_{2}, \mathcal{K}_{1}\right)$ by $\mathcal{T}(X)=A X-X B\left(X\right.$ in $\left.\mathcal{L}\left(\mathcal{F}_{2}, \mathcal{H}_{1}\right)\right)$.

LEMMA 2.5. If $\mathcal{T}$ is bounded below on $\mathcal{L}\left(\mathcal{F}_{2}, \mathcal{H}_{1}\right)$, then $A \oplus B$ (in $\left.\mathcal{L}\left(\mathcal{H}_{1} \oplus \mathcal{H}_{2}\right)\right)$ satisfies $(\mathrm{P})$ if and only if both $A$ and $B$ satisfy $(\mathrm{P})$.

Proof. We assume first that $A$ and $B$ satisfy (P). If $\left\{U_{n}\right\}$ is in $\delta(A \oplus B)$, we denote the operator matrix of $U_{n}$ by

$$
\left(\begin{array}{ll}
X_{n} & Y_{n} \\
Z_{n} & V_{n}
\end{array}\right) .
$$

Since $\lim \left\|U_{n}^{*}(A \oplus B) U_{n}-A \oplus B\right\|=0$, a calculation yields the relations

(1) $\lim \left\|X_{n}^{*} A X_{n}+Z_{n}^{*} B Z_{n}-A\right\|=0$,

(2) $\lim \left\|X_{n}^{*} A Y_{n}+Z_{n}^{*} B V_{n}\right\|=0$, and

(3) $\lim \left\|Y_{n}^{*} A Y_{n}+V_{n}^{*} B V_{n}-B\right\|=0$.

If we multiply (2) on the left by $X_{n}$, multiply (3) on the left by $Y_{n}$, and add the new limits, we obtain $\lim \left\|A Y_{n}-Y_{n} B\right\|=0$; since $\mathcal{T}$ is bounded below, it follows that $\lim \left\|Y_{n}\right\|=0$. Similarly, if we multiply (1) on the right by $Z_{n}^{*}$, 
multiply (2) on the right by $V_{n}^{*}$, and add the new limits, we obtain $\lim \left\|A Z_{n}^{*}-Z_{n}^{*} B\right\|=0$, whence $\lim \left\|Z_{n}^{*}\right\|=0$. Now

$$
\lim \left\|1-X_{n} X_{n}^{*}\right\|=\lim \left\|Y_{n} Y_{n}^{*}\right\|=0
$$

and

$$
\lim \left\|1-X_{n}^{*} X_{n}\right\|=\lim \left\|Z_{n}^{*} Z_{n}\right\|=0,
$$

and it follows that $X_{n}$ is invertible for all but finitely many values of $n$. A similar argument with $V_{n}$ allows us to assume that each $X_{n}$ and each $V_{n}$ is invertible.

If $X_{n}=R_{n}\left(X_{n}^{*} X_{n}\right)^{1 / 2}$ and $V_{n}=S_{n}\left(V_{n}^{*} V_{n}\right)^{1 / 2}$ denote, respectively, the polar decompositions of $X_{n}$ and $V_{n}$, then $R_{n}$ and $S_{n}$ are unitary; we will show that $\left\{R_{n}\right\}$ is in $\delta(A)$ and $\left\{S_{n}\right\}$ is in $\delta(B)$. Since $\lim \left\|1-X_{n}^{*} X_{n}\right\|=0$, the functional calculus implies that

(4) $\lim \left\|1-\left(X_{n}^{*} X_{n}\right)^{1 / 2}\right\|=0$.

Now

$$
\begin{aligned}
R_{n}^{*} A R_{n}-A= & R_{n}^{*} A R_{n}\left(1-\left(X_{n}^{*} X_{n}\right)^{1 / 2}\right) \\
& +\left(1-\left(X_{n}^{*} X_{n}\right)^{1 / 2}\right) R_{n}^{*} A R_{n}\left(X_{n}^{*} X_{n}\right)^{1 / 2}+X_{n}^{*} A X_{n}-A .
\end{aligned}
$$

Since $\lim \left\|Z_{n}^{*}\right\|=0$, the last equation, together with (1) and (4), imply that $\left\{R_{n}\right\}$ is in $\delta(A)$. Similarly, using (3), the fact that $\lim \left\|1-\left(V_{n}^{*} V_{n}\right)^{1 / 2}\right\|=0$, and $\lim \left\|Y_{n}\right\|=0$, it follows that $\left\{S_{n}\right\}$ is in $\delta(B)$.

Since $A$ and $B$ satisfy $(\mathrm{P})$, there exist sequences $\left\{P_{n}\right\} \subset \mathcal{U}\left(\mathcal{F C}_{1}\right)$ and $\left\{Q_{n}\right\} \subset \mathcal{Q}\left(\mathcal{F}_{2}\right)$ that re-implement, respectively, $\left\{R_{n}\right\}$ (for $A$ ) and $\left\{S_{n}\right\}$ (for $B)$. Since $P_{n} R_{n}^{*}$ commutes with $A$ and $Q_{n} S_{n}^{*}$ commutes with $B$, the unitary operator $U_{n}^{\prime}=\left(P_{n} R_{n}^{*} \oplus Q_{n} S_{n}^{*}\right) U_{n}$ satisfies $U_{n}^{\prime *}(A \oplus B) U_{n}^{\prime}=U_{n}^{*}(A \oplus B) U_{n}$. Since the matrix of $U_{n}^{\prime}$ is

$$
\left[\begin{array}{cc}
P_{n}\left(X_{n}^{*} X_{n}\right)^{1 / 2} & P_{n} R_{n}^{*} Y_{n} \\
Q_{n} S_{n}^{*} Z_{n} & Q_{n}\left(V_{n}^{*} V_{n}\right)^{1 / 2}
\end{array}\right],
$$

it follows that $\lim \left\|1-\mathrm{U}_{n}^{\prime}\right\|=0$, and therefore $A \oplus B$ satisfies (P).

For the converse, we assume that $A \oplus B$ satisfies (P) but that $A$ does not. If $\left\{W_{n}\right\}$ is an element of $\delta(A)$ that cannot be re-implemented, then $\left\{W_{n} \oplus\right.$ $1\}$ is in $\delta(A \oplus B)$, and thus may be re-implemented by a sequence $\left\{U_{n}\right\}$. We use the notation for the matrix of $U_{n}$ of the first part of the proof. A calculation yields the relations (1') $X_{n}^{*} A X_{n}+Z_{n}^{*} B Z_{n}-W_{n}^{*} A W_{n}=0,\left(2^{\prime}\right)$ $X_{n}^{*} A Y_{n}+Z_{n}^{*} B V_{n}=0$, and $\left(3^{\prime}\right) Y_{n}^{*} A Y_{n}+V_{n}^{*} B V_{n}-B=0$. Using $\left(1^{\prime}\right)-\left(3^{\prime}\right)$, calculations analogous to those above (using (1)-(3)) now yield $Z_{n}=0$ and $Y_{n}=0$. Thus, from $\left(1^{\prime}\right)$, it follows that $\left\{X_{n}\right\}$ re-implements $\left\{W_{n}\right\}$, which is a contradiction. A similar argument for $B$ completes the proof; we omit these details. 
THEOREM 2.6 If $\mathcal{T}$ is bounded below on $\mathcal{L}\left(\mathcal{F}_{2}, \mathcal{F}_{1}\right)$, then $A \oplus B$ (in $\mathcal{L}\left(\mathcal{F}_{1} \oplus\right.$ $\left.F_{2}\right)$ ) has a local cross-section if and only if $A$ and $B$ have local cross-sections.

Proof. Let $\delta>0$ be such that $\|A X-X B\| \geqslant \delta\|X\|$ for each $X$ in $\mathcal{L}\left(\mathcal{F G}_{2}, \mathcal{H}_{1}\right)$. Let $\varepsilon>0$ be chosen such that $A$ and $B$ have local cross-sections of the form $\left(\varphi_{A}, \mathscr{B}(A, \varepsilon)\right)$ and $\left(\varphi_{B}, \mathscr{B}(B, \varepsilon)\right)$ respectively. Let $U$ be a unitary operator on $\mathcal{F}_{1} \oplus \mathcal{F}_{2}$ with operator matrix $U=\left(\begin{array}{l}X \\ Z\end{array}\right)$, and let $X=$ $R\left(X^{*} X\right)^{1 / 2}$ and $V=S\left(V^{*} V\right)^{1 / 2}$ denote the respective polar decompositions of $X$ and $V$. We will show first that there exists $\varepsilon_{0}>0$ such that if

$$
\left\|U^{*}(A \oplus B) U-(A \oplus B)\right\|<\varepsilon_{0},
$$

then $S$ and $R$ are unitary, $\left\|R^{*} A R-A\right\|<\varepsilon$, and $\left\|S^{*} B S-B\right\|<\varepsilon$.

If (*) is satisfied for some $\varepsilon_{0}>0$, then we have (1) $\left\|X^{*} A X+Z^{*} B Z-A\right\|$ $<\varepsilon_{0}$, (2) $\left\|X^{*} A Y+Z^{*} B V\right\|<\varepsilon_{0}$, and (3) $\left\|Y^{*} A Y+V^{*} B V-B\right\|<\varepsilon_{0}$. Now

$$
\begin{aligned}
\|Y\| & <(1 / \delta)\|A Y-Y B\| \\
& =(1 / \delta)\left\|\left(X X^{*}+Y Y^{*}\right) A Y+\left(X Z^{*}+Y V^{*}\right) B V-Y B\right\| \\
& =(1 / \delta)\left\|X\left(X^{*} A Y+Z^{*} B V\right)+Y\left(Y^{*} A Y+V^{*} B V-B\right)\right\|<2 \varepsilon_{0} / \delta .
\end{aligned}
$$

Similarly, we have $\|Z\|<2 \varepsilon_{0} / \delta$. If we set $K=4 \varepsilon_{0}^{2} / \delta^{2}$, then $\left\|1-X X^{*}\right\|=$ $\left\|Y Y^{*}\right\|=\|Y\|^{2}<K$; likewise $\left\|1-X^{*} X\right\|,\left\|1-V V^{*}\right\|$, and $\left\|1-V^{*} V\right\|$ are less than $K$. Thus if $K<1$ (i.e., if $\varepsilon_{0}<\delta / 2$ ), then $V$ and $X$ are invertible and therefore $R$ and $S$ are unitary. If we assume also that $\varepsilon_{0}<1$, then

$$
\begin{aligned}
\left\|R^{*} A R-A\right\| \leqslant & \left\|R^{*} A R\left(1-\left(X^{*} X\right)^{1 / 2}\right)\right\| \\
& +\left\|\left(1-\left(X^{*} X\right)^{1 / 2}\right) R^{*} A R\left(X^{*} X\right)^{1 / 2}\right\| \\
& +\left\|Z^{*} B Z\right\|+\left\|X^{*} A X+Z^{*} B Z-A\right\| \\
< & 2\|A\|\left(1-(1-K)^{1 / 2}\right)+\|B\|\|Z\|^{2}+\varepsilon_{0} \\
< & K(2\|A\|+\|B\|)+\varepsilon_{0}<\varepsilon_{0}\left(\left(4 / \delta^{2}\right)(2\|A\|+\|B\|)+1\right) \\
& =\varepsilon_{0}\left(8\|A\|+4\|B\|+\delta^{2}\right) / \delta^{2} .
\end{aligned}
$$

We may estimate $\left\|S^{*} B S-B\right\|$ similarly, and it follows that if $(*)$ is satisfied for

$$
0<\varepsilon_{0}<\min \left\{1, \frac{\delta}{2}, \frac{\varepsilon \delta^{2}}{8\|A\|+4\|B\|+\delta^{2}}, \frac{\varepsilon \delta^{2}}{8\|B\|+4\|A\|+\delta^{2}}\right\},
$$

then $R$ and $S$ are unitary, $\left\|R^{*} A R-A\right\|<\varepsilon$, and $\left\|S^{*} B S-B\right\|<\varepsilon$.

For $U^{*}(A \oplus B) U$ in $\mathscr{B}\left(A \oplus B, \varepsilon_{0}\right)$, we now define $\varphi\left(U^{*}(A \oplus B) U\right)=$ $\left(\varphi_{A}\left(R^{*} A R\right) R^{*} \oplus \varphi_{B}\left(S^{*} B S\right) S^{*}\right) U$, and we will prove that $\left(\varphi, \mathscr{B}\left(A \oplus B, \varepsilon_{0}\right)\right)$ is a local cross-section. To prove that $\varphi$ is well defined, suppose that $U^{*}(A \oplus B) U=U_{1}^{*}(A \oplus B) U_{1}$ for $U_{1}$ in $\left.\vartheta_{\left(\mathcal{C}_{1}\right.} \oplus \mathcal{F}_{2}\right)$. Since $\mathcal{T}$ is bounded 
below and $U_{1} U^{*}$ commutes with $A \oplus B$, it follows that $U_{1} U^{*}=L \oplus M$, where $L$ and $M$ are unitary operators that commute with $A$ and $B$ respectively. Thus the operator matrix of $U_{1}=(L \oplus M) U$ is $\left(\begin{array}{cc}L X & L Y \\ M V\end{array}\right)$. Now the respective polar decompositions of $L X$ and $M V$ are $L X=L R\left(X^{*} X\right)^{1 / 2}$ and $M V=M S\left(V^{*} V\right)^{1 / 2}$, and we have

$$
\begin{aligned}
\left(\varphi_{A}\left((L R)^{*} A L R\right) R^{*} L^{*} \oplus \varphi_{B}\left((M S)^{*} B M S\right) S^{*} M^{*}\right) U_{1} \\
\quad=\left(\varphi_{A}\left(R^{*} A R\right) R^{*} \oplus \varphi_{B}\left(S^{*} B S\right) S^{*}\right)\left(L^{*} \oplus M^{*}\right)\left(\begin{array}{cc}
L X & L Y \\
M Z & M V
\end{array}\right) \\
\quad=\varphi\left(U^{*}(A \oplus B) U\right),
\end{aligned}
$$

which completes the proof that $\varphi$ is well defined. Since $\varphi_{A}\left(R^{*} A R\right) R^{*}$ commutes with $A$ and $\varphi_{B}\left(S^{*} B S\right) S^{*}$ commutes with $B$, it is clear that $\varphi$ is a cross-section, and it is also clear that $\varphi(A \oplus B)=1$. To complete the proof it suffices to prove that $\varphi$ is continuous. Suppose that $\left\{U_{n}\right\}_{n=0}^{\infty} \subset \mathcal{Q}\left(\mathcal{F}_{1} \oplus \mathcal{F C}_{2}\right)$ is a sequence such that $U_{n}^{*}(A \oplus B) U_{n} \rightarrow U_{0}^{*}(A \oplus B) U_{0}$ in $\mathscr{B}\left(A \oplus B, \varepsilon_{0}\right)$. Since $A$ and $B$ satisfy (P), Lemma 2.5 implies that there is a sequence $\left\{W_{n}\right\}_{n=1}^{\infty} \subset \mathcal{U}\left(\mathcal{H}_{1} \oplus \mathcal{H}_{2}\right)$ such that $W_{n} \rightarrow 1$ and such that $W_{n}^{*}(A \oplus B) W_{n}=$ $U_{0} U_{n}^{*}(A \oplus B) U_{n} U_{0}^{*}$ for each $n$. Thus

$$
U_{n}^{*}(A \oplus B) U_{n}=U_{0}^{*} W_{n}^{*}(A \oplus B) W_{n} U_{0},
$$

and since $\varphi$ is well defined, we may verify the continuity of $\varphi$ using the sequence $\left\{W_{n} U_{0}\right\}$; moreover, the limit

$$
\varphi\left(U_{0}^{*} W_{n}^{*}(A \oplus B) W_{n} U_{0}\right) \rightarrow \varphi\left(U_{0}^{*}(A \oplus B) U_{0}\right)
$$

is now a simple consequence of the definition of $\varphi$ and the fact that $W_{n} U_{0} \rightarrow U_{0}$.

For the converse, suppose that $A \oplus B$ has a cross-section of the form $(\varphi, \mathscr{B}((A \oplus B), \varepsilon))$ for some $\varepsilon>0$. If $X$ is in $2\left(\mathcal{F}_{1}\right)$ and $\left\|X^{*} A X-A\right\|<\varepsilon$, then $\varphi\left(X^{*} A X \oplus B\right)\left(X^{*} \oplus 1\right)$ commutes with $A \oplus B$ and therefore is of the form $Z \oplus W$, where $Z$ commutes with $A$. We now define $\varphi_{A}\left(X^{*} A X\right)=Z X$ and it follows easily that $\varphi_{A}$ is well defined, that $\varphi_{A}$ is a cross-section, and that $\varphi_{A}(A)=1$. To complete the proof we will prove that $\varphi_{A}$ is continuous on $\mathscr{B}(A, \varepsilon)$. Suppose $\left\{X_{n}\right\}_{n=0}^{\infty} \subset \mathcal{U}\left(\mathcal{H}_{1}\right)$ is a sequence such that $X_{n}^{*} A X_{n} \rightarrow$ $X_{0}^{*} A X_{0}$ in $\mathscr{B}(A, \varepsilon)$. Since $A \oplus B$ satisfies (P), Lemma 2.5 implies that there exists a sequence $\left\{W_{n}\right\} \subset \mathcal{U}\left(\mathcal{F}_{1}\right)$ such that $W_{n} \rightarrow 1$ and such that $W_{n}^{*} A W_{n}$ $=X_{0} X_{n}^{*} A X_{n} X_{0}^{*}$ for each $n$. Since $X_{n}^{*} A X_{n}=X_{0}^{*} W_{n}^{*} A W_{n} X_{0}$ and since $\varphi_{A}$ is well defined, we may use the sequence $\left\{W_{n} X_{0}\right\}$ in calculating $\varphi_{A}\left(X_{n}^{*} A X_{n}\right)$. It now follows readily from the definition of $\varphi_{A}$ and from the fact that $W_{n} X_{0} \rightarrow X_{0}$ that $\varphi_{A}\left(X_{n}^{*} A X_{n}\right)=\varphi_{A}\left(X_{0}^{*} W_{n}^{*} A W_{n} X_{0}\right) \rightarrow \varphi_{A}\left(X_{0}^{*} A X_{0}\right)$, and the proof is complete.

Let $n$ be an integer greater than or equal to 2. Let $A_{i}$ be in $\mathcal{L}\left(\mathcal{F C}_{i}\right)$, 
$i=1, \ldots, n$, and suppose that $\sigma\left(A_{i}\right) \cap \sigma\left(A_{j}\right)=\varnothing$ for $i \neq j$. Let $B=A_{1}$ $\oplus \cdots \oplus A_{n}$ in $\mathcal{L}\left(\mathcal{F}_{1} \oplus \cdots \oplus \mathcal{H}_{n}\right)$.

THEOREM 2.7. $B$ satisfies $(\mathrm{P})$ if and only if $A_{i}$ satisfies $(\mathrm{P}), i=1, \ldots, n$.

PRoof. Let $n=2$ and define $\mathscr{T}(X)=A_{1} X-X A_{2}$ for $X$ in $\mathcal{L}\left(\mathcal{F G}_{2}, \mathcal{G G}\right)$. Rosenblum's theorem [7, p. 8] implies that $\mathcal{G}$ is invertible, and the result follows from Lemma 2.5. The proof may now be completed by a straightforward induction on $n$.

By using Theorem 2.6 and an induction proof like that in Theorem 2.7, we obtain the following result.

THEOREM 2.8. $B$ has a local cross-section if and only if $A_{i}$ has a local cross-section, $i=1, \ldots, n$.

3. Normal operators and isometries. In this section we prove that if an operator is normal or isometric, then it satisfies $(\mathrm{P})$ if and only if its spectrum is finite, in which case it also has a local cross-section.

THEOREM 3.1. A normal operator $N$ in $\mathfrak{L}(\mathcal{F C})$ satisfies $(\mathrm{P})$ if and only if its spectrum is finite.

Proof. If $\sigma(N)$ is finite, then $N$ is unitarily equivalent to a finite direct sum of distinct complex scalars, so the result follows from Theorem 2.6.

If $\sigma(N)$ is infinite, there exists a convergent sequence $\left\{\lambda_{n}\right\} \subset \sigma(N)$ and a sequence $\left\{D_{n}\right\}$ of open disks such that (1) $D_{n}$ is centered at $\lambda_{n}$ with radius $r_{n}$, (2) $\lim r_{n}=0$, and (3) $D_{n}^{-} \cap D_{m}^{-}=\varnothing$ for $n \neq m$. Let $E$ denote the spectral measure of $N$. Since $D_{n}$ is open and $D_{n} \cap \sigma(N) \neq \varnothing$, then $E_{n}=E\left(D_{n}\right) \neq 0$ (see [7, Theorem 1.13, p. 18]), and we let $e_{n}$ denote a unit vector in the range of $E_{n}$. Condition (3) implies that we may extend $\left\{e_{n}\right\}$ to an orthonormal basis $\left\{e_{n}\right\} \cup\left\{f_{m}\right\}$ for $\mathcal{H C}$ (where the $f_{m}$ 's will be absent in some cases). We define a sequence $\left\{U_{n}\right\} \subset \mathcal{U}(\mathcal{H C})$ as follows: $U_{n}\left(e_{j}\right)=e_{j}(j \neq n, n+1), U_{n}\left(e_{n}\right)=$ $e_{n+1}, U_{n}\left(e_{n+1}\right)=e_{n}$, and $U_{n}\left(f_{m}\right)=f_{m}$ (all $m$ ). We have

$$
\begin{aligned}
&\left\|N U_{n}^{*} e_{n}-U_{n}^{*} N e_{n}\right\| \\
&<\left\|\left(N-\lambda_{n+1}\right) U_{n}^{*} e_{n}\right\|+\left\|\left(\lambda_{n+1}-\lambda_{n}\right) U_{n}^{*} e_{n}\right\|+\left\|\lambda_{n} U_{n}^{*} e_{n}-U_{n}^{*} N e_{n}\right\| \\
&<\left\|\left(N-\lambda_{n+1}\right) E_{n+1} e_{n+1}\right\|+\left|\lambda_{n+1}-\lambda_{n}\right|+\left\|\left(N-\lambda_{n}\right) E_{n} e_{n}\right\| \\
&<r_{n+1}+\left|\lambda_{n+1}-\lambda_{n}\right|+r_{n} .
\end{aligned}
$$

If $x$ is in $E_{n} \mathcal{H C} \Theta\left\langle e_{n}\right\rangle$, then

$$
\begin{aligned}
\left\|N U_{n}^{*} x-U_{n}^{*} N x\right\| & \leqslant\left\|N U_{n}^{*} x-\lambda_{n} U_{n}^{*} x\right\|+\left\|\lambda_{n} U_{n}^{*} x-U_{n}^{*} N x\right\| \\
& <2\left\|\left(N-\lambda_{n}\right) E_{n} x\right\|<2 r_{n}\|x\| .
\end{aligned}
$$

Similarly, we have 


$$
\begin{gathered}
\left\|\left(N U_{n}^{*}-U_{n}^{*} N\right) e_{n+1}\right\|<r_{n}+\left|\lambda_{n}-\lambda_{n+1}\right|+r_{n+1} \text { and } \\
\left\|N U_{n}^{*} x-U_{n}^{*} N x\right\|<2 r_{n+1}\|x\|
\end{gathered}
$$

for $x$ in $E_{n+1} \mathcal{H} \Theta\left\langle e_{n+1}\right\rangle$. Since $N U_{n}^{*}-U_{n}^{*} N=0$ on $\left(E_{n} \mathcal{H} \oplus E_{n+1} \mathcal{H}\right)^{\perp}$, these relations imply that

$$
\left\|U_{n}^{*} N U_{n}-N\right\|=\left\|U_{n}^{*} N-N U_{n}^{*}\right\| \leqslant 2\left(2 \dot{r}_{n}+2 r_{n+1}+\left|\lambda_{n+1}-\lambda_{n}\right|\right),
$$

and therefore $\left\{U_{n}\right\}$ is in $\delta(N)$. If $W$ is a unitary operator such that $W^{*} N W=U_{n}^{*} N U_{n}$, then $W U_{n}^{*}$ commutes with $N$ and thus also with $E_{n+1}$ (see [7, Fuglede's Theorem, p. 19]). We have $W e_{n}=W U_{n}^{*} E_{n+1} e_{n+1}=$ $E_{n+1} W U^{*} e_{n+1}$, and (3) now implies that $\left(W e_{n}, e_{n}\right)=0$. It follows that if $|\lambda|=1$, then $\|W-\lambda\|>2^{1 / 2}$, which completes the proof.

If $M$ and $N$ are in $\mathcal{L}(\mathcal{H})$, we denote by $\mathcal{Q}(N, M)$ the norm closed subalgebra of $\mathcal{L}(\mathcal{H})$ generated by $N, M$, and 1 .

THEOREM 3.2. If $N$ is a normal operator with finite spectrum, then $\pi_{N}$ has a local cross-section $\left(\varphi_{N}, \mathscr{B}\right)$ such that for each $M$ in $\mathscr{B}, \varphi_{N}(M)$ is in $\mathcal{Q}(N, M)$.

Proof. We first give the proof for a projection $P$ in $\mathcal{L}(\mathcal{H})$. Let $\mathscr{B}=\{Q$ in $\mathscr{U}(P) \mid\|P-Q\|<1\}$, and let $Q=\pi_{P}(U)$ be in $\mathscr{B}$. With respect to the decomposition $\mathscr{H}=(\operatorname{ker}(P))^{\perp} \oplus \operatorname{ker}(P)$, we denote the matrix of $U$ by $\left(\begin{array}{ll}X & Y \\ Z & V\end{array}\right)$; thus the matrix of $Q$ is

$$
\left(\begin{array}{cc}
X^{*} X & X^{*} Y \\
Y^{*} X & Y^{*} Y
\end{array}\right)
$$

Since $\|P U-U P\|=\left\|U^{*} P U-P\right\|=\|Q-P\|<1$, a matrix calculation of $P U-U P$ shows that $\|Z\|<1$ and $\|Y\|<1$. Now $\left\|1-V^{*} V\right\|=\left\|Y^{*} Y\right\|$ $=\|Y\|^{2}<1$, and similarly $\left\|1-X X^{*}\right\|=\left\|Y Y^{*}\right\|<1,\left\|1-V V^{*}\right\|=\left\|Z Z^{*}\right\|$ $<1$, and $\left\|1-X^{*} X\right\|=\left\|Z^{*} Z\right\|<1$; thus, the polar decomposition implies that $X$ and $V$ are invertible. In particular, $X^{*} X$ and $1-Y^{*} Y=V^{*} V$ are invertible, and we define $\varphi_{P}(Q)$ by the matrix

$$
\left[\begin{array}{cc}
\left(X^{*} X\right)^{1 / 2} & \left(X^{*} X\right)^{-1 / 2} X^{*} Y \\
-\left(1-Y^{*} Y\right)^{-1 / 2} Y^{*} X & \left(1-Y^{*} Y\right)^{1 / 2}
\end{array}\right]
$$

Now $\varphi_{P}(Q)$ is independent of the choice of $U$ since it is defined in terms of the matrix components of $Q$. Thus $\varphi_{P}(Q)$ is well defined and it follows from the identity

$$
\varphi_{P}(Q)=\left(\left(X^{*} X\right)^{-1 / 2} X^{*} \oplus\left(V^{*} V\right)^{-1 / 2} V^{*}\right) U
$$

that $\varphi_{P}(Q)$ is unitary, that $\varphi_{P}(P)=1$ and that $\pi_{P}\left(\varphi_{P}(Q)\right)=Q$. It is clear from the matrix of $Q$ that $\varphi_{P}$ is continuous on $\mathscr{B}$. That $\varphi_{P}(Q)$ is in $Q(P, Q)$ follows from the identity 


$$
\begin{aligned}
\varphi_{P}(Q)= & (P Q P)^{1 / 2}+(1-P+P Q P)^{-1 / 2} P Q(1-P) \\
& +((1-P)(1-Q(1-P)))^{1 / 2} \\
& -(P+(1-P)(1-Q(1-P)))^{-1 / 2}(1-P) Q P .
\end{aligned}
$$

Indeed, the Weierstrass approximation theorem and the functional calculus for positive operators imply that each term in the preceding sum is in $\mathbb{Q}(P, Q)$.

If $\sigma(N)=\left\{\lambda_{1}, \ldots, \lambda_{n}\right\}$, then $N$ is diagonalizable, and thus there exist polynomials $p_{1}(z), \ldots, p_{n}(z)$, such that $p_{1}(N), \ldots, p_{n}(N)$ are pairwise orthogonal projections and such that $\sum_{i=1}^{n} p_{i}(N)=1$ and $N=\sum_{i=1}^{n} \lambda_{i} p_{i}(N)$. Let $\delta>0$ be a positive number such that if $M$ is in $Q(N)$ and $\|M-N\|<\delta$, then $\left\|p_{i}(M)-p_{i}(N)\right\|<1, i=1, \ldots, n$. Let $\mathscr{B}=\{M$ in $2(N) \mid\|M-N\|$ $<\delta\}$; thus, if $M$ is in $\mathscr{B}$, then for each fixed $i, p_{i}(M)$ is in the domain of the local cross-section $\left(\varphi_{i}, \mathfrak{B}_{i}\right)$ for $p_{i}(N)$ that was defined above. We now define $\varphi_{N}(M)=\sum_{i=1}^{n} p_{i}(N) \varphi_{i}\left(p_{i}(M)\right) p_{i}(M)$. It is clear that $\varphi_{N}(M)$ is in $\mathbb{Q}(N, M)$ and that $\varphi_{N}$ is continuous on $\mathscr{B}$. Since for each $i$ we have $\varphi_{i}\left(p_{i}(M)\right)^{*} p_{i}(N) \varphi_{i}\left(p_{i}(M)\right)=p_{i}(M)$, a calculation shows that $\varphi_{N}(M)$ is a unitary operator and that $\varphi_{N}(N)=1$ and $\pi_{N}\left(\varphi_{N}(M)\right)=M$. Thus $\left(\varphi_{N}, \mathscr{B}\right)$ is a cross-section for $N$ and the proof is complete.

Let $\mathscr{Q}$ denote a $C^{*}$-subalgebra of $\mathcal{L}(\mathcal{H})$ and let $\mathscr{Q}(\mathbb{Q})=\mathscr{Q}(\mathcal{H}) \cap \mathbb{Q}$. For $T$ in $\mathscr{Q}$, let $\mathscr{Q}_{\mathscr{Q}}(T)$ denote the $\mathscr{Q}(\mathscr{Q})$ orbit of $T$ in $\mathscr{Q}$ and define $\pi(\mathscr{Q})_{T}$ : $\mathcal{U}(\mathbb{Q}) \rightarrow \mathcal{Q}_{\mathbb{Q}}(T)$ by $\pi(\mathbb{Q})_{T}(U)=U^{*} T U$.

COROLlaRY 3.3. If $N$ is a normal operator in $\mathbb{Q}$ with finite spectrum, then $\pi(\mathfrak{Q})_{N}$ has a local cross-section.

REMARK. In the case $\mathbb{Q}=\mathscr{L}(\mathcal{H})$, Corollary 3.3 is a direct consequence of Theorem 2.8. Note also that since any two projections differ in norm by at most 1, then the cross-section given in Theorem 3.2 for a projection $P$ is densely defined in $थ(P)$; however, it is easy to prove that this cross-section cannot be extended to all of $U(P)$. In [2] it was proven that if $T$ has a cross-section, then each path in $\mathcal{U}(T)$ can be lifted to a path in $U(\mathcal{H C})$. By adapting the argument used in proving Theorem 3.1, it is not difficult to prove that there exists a diagonalizable normal operator (with infinite spectrum) which does not enjoy this path lifting property.

In the sequel $\mathcal{K}_{1}$ and $\mathscr{F}_{2}$ denote separable complex Hilbert spaces and $\mathscr{K}=\mathcal{K}_{1} \oplus \mathcal{K}_{2}$.

COROLLARY 3.4. If $N$ is a normal operator in $\mathcal{L}\left(\mathcal{H C}_{1}\right)$ with infinite spectrum and if $T$ is in $\mathcal{L}\left(\mathcal{H}_{2}\right)$, then $N \oplus T$ does not satisfy (P).

Proof. We use the notation of the proof of Theorem 3.1. Suppose that $N \oplus T$ does satisfy (P) and let $\left\{W_{n}\right\} \subset \mathcal{Q}(\mathcal{K})$ denote a sequence that 
re-implements $\left\{U_{n} \oplus 1\right\}$ for $N \oplus T$. Now $V_{n}=W_{n}\left(U_{n}^{*} \oplus 1\right)$ commutes with $N \oplus T$, and if we denote the matrix of $V_{n}$ by

$$
\left(\begin{array}{ll}
A_{n} & B_{n} \\
C_{n} & D_{n}
\end{array}\right),
$$

then a calculation shows that $A_{n}$ commutes with $N$. Fuglede's Theorem [7, Theorem 1.13] implies that $A_{n}$ commutes with $E_{n+1}$ and thus $A_{n} U_{n} e_{n}=$ $A_{n} E_{n+1} e_{n+1}=E_{n+1} A_{n} e_{n+1}$; in particular $\left(A_{n} U_{n} e_{n}, e_{n}\right)=0$. Now $\left\|A_{n} U_{n}-1\right\|$ $>\left|\left(\left(A_{n} U_{n}-1\right) e_{n}, e_{n}\right)\right|=1$; however, since $V_{n}\left(U_{n} \oplus 1\right)=W_{n} \rightarrow 1$, a matrix calculation shows that $A_{n} U_{n} \rightarrow 1$, which is a contradiction.

If $T$ is in $\mathcal{L}(\mathcal{F C})$, let $R_{e}(T)$ denote the set of all reducing essential eigenvalues of $T$ (see [8]).

LEMMA 3.5. Let $T$ be in $\mathcal{E}(\mathcal{F C})$ and suppose $R_{e}(T)$ is nonempty. Then there exists a sequence $\left\{U_{n}\right\}$ in $\delta(T)$ such that for each $n$, $\inf _{|\lambda|=1}\left\|U_{n}-\lambda\right\| \geqslant 2^{1 / 2}$.

Proof. Let $\lambda$ be in $R_{e}(T)$. Corollary 4.9 of [8] implies that there exists a sequence $\left\{W_{n}\right\}$ of unitary operators from $\mathcal{H}$ onto $\mathcal{H} \oplus \mathscr{H}$ such that

$$
W_{n}^{*}(T \oplus \lambda) W_{n} \rightarrow T \text {. }
$$

Let $V$ be a unitary operator on $\mathcal{H}$ such that $\inf _{|\lambda|=1}\|V-\lambda\| \geqslant 2^{1 / 2}$ and let $U_{n}=W_{n}^{*}(1 \oplus V) W_{n}$. Then

$$
\begin{aligned}
\left\|U_{n}^{*} T U_{n}-T\right\| \leqslant & \left\|U_{n}^{*}\left(T-W_{n}^{*}(T \oplus \lambda) W_{n}\right) U_{n}\right\| \\
& +\left\|U_{n}^{*} W_{n}^{*}(T \oplus \lambda) W_{n} U_{n}-T\right\| \\
& =2\left\|T-W_{n}^{*}(T \oplus \lambda) W_{n}\right\| \rightarrow 0 .
\end{aligned}
$$

Therefore, $\left\{U_{n}\right\}$ satisfies the required conditions, and the proof is complete.

LEMMA 3.6. Let $V$ be an irreducible operator in $\mathcal{L}\left(\mathcal{H}_{1}\right)$. Suppose there exist a sequence $\left\{U_{n}\right\}$ in $\delta(V)$ and a positive number $\varepsilon$ such that for each $n$, $\inf _{|\lambda|=1}\left\|U_{n}-\lambda\right\| \geqslant \varepsilon$. Then for each $T$ in $\mathcal{L}\left(\mathcal{F}_{2}\right), V \oplus T$ does not satisfy (P).

Proof. If $V \oplus T$ does satisfy $(\mathrm{P})$, then there exists a sequence $\left\{W_{n}\right\} \subset$ $\mathcal{U}(\mathcal{K})$ that re-implements $\left\{U_{n} \oplus 1\right\}$ for $V \oplus T$. If we denote the operator matrix of $\left(U_{n} \oplus 1\right) W_{n}^{*}$ by

$$
\left(\begin{array}{ll}
A_{n} & B_{n} \\
C_{n} & D_{n}
\end{array}\right),
$$

then since $W_{n}^{*} \rightarrow 1$, a calculation of $W_{n}^{*}=\left(U_{n}^{*} \oplus 1\right)\left(U_{n} \oplus 1\right) W_{n}^{*}$ shows that $U_{n}^{*} A_{n} \rightarrow 1$ and $C_{n} \rightarrow 0$. Since $\left(U_{n} \oplus 1\right) W_{n}^{*}$ commutes with $V \oplus T$ and $(V \oplus T)^{*}, A_{n}$ commutes with $V$ and $V^{*}$, and the irreducibility of $V$ implies that $A_{n}=\lambda_{n}$. Now $\left.\lim || \lambda_{n}\right|^{2}-1 \mid=\lim \left\|C_{n}^{*} C_{n}\right\|=0$, and thus $\left|\lambda_{n}\right| \rightarrow 1$. Since there exists a convergent subsequence $\lambda_{n_{k}} \rightarrow \lambda(|\lambda|=1)$, it follows that 
$\lim \left\|U_{n_{k}}-\lambda\right\|=0$, which is a contradiction.

The preceding two lemmas now yield the following result.

THEOREM 3.5. If $V$ is an irreducible operator in $\mathcal{L}\left(\mathcal{F C}_{1}\right)$ and $R_{e}(V)$ is nonempty, then for each operator $T$ in $\mathcal{L}\left(\mathcal{F G}_{2}\right), V \oplus T$ does not satisfy $(\mathrm{P})$.

EXAMPLE 3.6. Using the preceding theorem we will show that there exist compact quasi-nilpotent operators that do not satisfy (P). Indeed, if $K$ is compact, then 0 is in $R_{e}(K)$, and thus if $K$ is irreducible, then $K$ does not satisfy (P). One example of this type is the Volterra integral operator (see [7, Proposition 4.11, p. 82]). Let $\left\{e_{n}\right\}_{n=-\infty}^{+\infty}$ denote an orthonormal basis for $\mathcal{H}$, and let $\left\{\lambda_{n}\right\}_{n=-\infty}^{+\infty}$ denote a sequence of nonzero complex numbers. We define an injective bilateral shift $W$ by $W e_{n}=\lambda_{n} e_{n+1}$. If $W$ is compact (or, equivalently, if $\lim _{n \rightarrow \infty} \lambda_{n}=\lim _{n \rightarrow-\infty} \lambda_{n}=0$ ), then $W$ is quasi-nilpotent (see [5, p. 48]), and since the sequence $\left\{\lambda_{n}\right\}$ is nonperiodic, it follows from [5, Problem 129] that $W$ is irreducible. Therefore each injective bilateral weighted shift that is compact does not satisfy $(\mathrm{P})$.

COROLLARY 3.6. If $V$ has a cross-section and $R_{e}(V)$ is nonempty, then $V$ has a nontrivial reducing subspace.

COROLlaRY 3.7. If $V$ is an irreducible hyponormal operator in $\mathcal{L}\left(\mathcal{F C}_{1}\right)$ $\left(\operatorname{dim} \mathcal{F}_{1}>1\right)$, then for each operator $T$ in $\mathcal{L}\left(\mathcal{F}_{2}\right), V \oplus T$ does not satisfy $(\mathrm{P})$.

Proof. If $V$ is a hyponormal operator in $\mathcal{E}\left(\mathcal{F}_{1}\right)$ and $\mathscr{H}_{1}$ is finite dimensional, then $V$ is normal, and thus if $\operatorname{dim}\left(\mathcal{F}_{1}\right)>1$, then $V$ is reducible. We may therefore assume that $\operatorname{dim}\left(\mathcal{F C}_{1}\right)=\aleph_{0}$. Theorem 3.10 of [8] implies that if $V$ is hyponormal, then $R_{e}(V)$ is nonempty. The result now follows from Theorem 3.5.

REMARK. The preceding result applies in particular if $V$ is a unilateral shift of multiplicity one. If $\|T\|<1$, the conclusion that $V \oplus T$ does not satisfy (P) may be obtained from Lemma 2.5. Indeed, for $\|T\|<1$, it is not difficult to prove that the operator $\tau$ on $\mathcal{L}\left(\mathcal{F}_{2}, \mathcal{F C}_{1}\right)$ defined by $\tau(X)=V X-X T(X$ in $\left.\mathcal{L}\left(\mathcal{F C}_{2}, \mathcal{F C}_{1}\right)\right)$ is left invertible (although not invertible) in $\mathcal{L}\left(\mathcal{L}\left(\mathcal{F}_{2}, \mathcal{F C}_{1}\right)\right)$ and is thus bounded below.

The preceding corollary implies that if a hyponormal operator satisfies $(\mathrm{P})$, then it has no minimal infinite dimensional reducing subspace. We conjecture that a hyponormal operator satisfies $(\mathrm{P})$ if and only if its spectrum is finite.

COROLlaRY 3.8. An isometry $V$ in $\mathcal{L}(\mathcal{H C})$ satisfies (P) if and only if its spectrum is finite, in which case $V$ has a local cross-section.

Proof. If $V$ is unitary, the result follows from Theorem 3.1 and Theorem 3.2. If $V$ is nonunitary, the von Neumann decomposition implies that $V$ has a unilateral shift of multiplicity one as a direct summand. Corollary 3.7 now 
implies that $V$ does not satisfy $(\mathrm{P})$, and since the spectrum of each nonunitary isometry is infinite, the proof is complete.

4. Nonnormal operators with cross-sections. In this section we give examples of nonnormal operators with cross-sections. Let $T$ be an operator in $\mathcal{L}(\mathcal{H C})$ with closed range and let $C^{*}(T)$ denote the $C^{*}$-subalgebra of $\mathcal{L}(\mathcal{F C})$ generated by $T$. We will make use of the following simple observations: If $T=U P$ denotes the polar decomposition of $T$, then $P$ is in $C^{*}(T)$; if $\left\{U_{n}\right\}_{n=0}^{\infty} \subset$ $Q(\mathcal{H C})$ and $U_{n}^{*} T U_{n} \rightarrow U_{0}^{*} T U_{0}$, then $U_{n}^{*} S U_{n} \rightarrow U_{0}^{*} S U_{0}$ for each $S$ in $C^{*}(T)$; if $T$ commutes with $U_{0}$, then $S$ commutes with $U_{0}$ for each $S$ in $C^{*}(T)$. Let $P=0 \oplus 1$ in $\mathcal{L}(\mathcal{H} \oplus \mathcal{H})$; in the sequel $(\varphi, \mathscr{B}(P, 1))$ will denote a local cross-section for $P$ (cf. Theorem 3.2). If $T$ and $S$ are in $\mathcal{L}(\mathcal{H C}), S \neq 0$, then $M \equiv M(T, S)$ denotes the nonnormal operator on $\mathcal{H C} \oplus \mathcal{H}$ whose operator matrix is $\left(\begin{array}{c}T \\ S\end{array}\right)$.

LEMma 4.1. Let $T$ and $S$ be in $\mathcal{L}(\mathcal{H C})$ and suppose that $T^{*} T+S^{*} S$ is invertible. If $\varepsilon>0$, then there exists $\delta>0$ such that if $X$ is in $\mathscr{B}(M, \delta)$, then (i) $\left\|P_{\operatorname{ker}(X)}-P\right\|<1$ and (ii) $\left\|V X V^{*}-M\right\|<\varepsilon$, where $V=\varphi\left(P_{\operatorname{ker}(X)}\right)$.

Proof. Let $f$ be a real continuous function on $[0,\|M\|]$. It follows from the functional calculus that the mapping $F: \mathcal{Q}(M) \rightarrow \mathscr{L}(\mathcal{F C})$ defined by $F(X)=$ $f\left(\left(X^{*} X\right)^{1 / 2}\right)$ is norm continuous. Since $M$ has closed range, 0 is an isolated point of $\sigma\left(\left(M^{*} M\right)^{1 / 2}\right)$, and thus the characteristic function of $\{0\}$ with respect to $\sigma\left(\left(M^{*} M\right)^{1 / 2}\right)$ can be extended to a function $f$ that is continuous on $[0,\|M\|]$. Now $F(X)=P_{\text {ker }(X)}$ for each $X$ in $\mathcal{Q}(M)$, and since $F$ is continuous, there exists $\delta_{1}>0$ such that if $\|X-M\|<\delta_{1}(X$ in $U(M)$ ), then $\left\|P_{\text {ker }(X)}-P\right\|<1$. Thus $V=\varphi\left(P_{\operatorname{ker}(X)}\right)$ is defined and

$$
\left\|V X V^{*}-M\right\|<2\|V-1\|\|M\|+\|X-M\| \text {. }
$$

Since $\varphi$ is continuous, there exists $\delta<\min \left\{\varepsilon / 2, \delta_{1}\right\}$ such that if $X$ is in $\mathscr{B}(M, \delta)$, then $\left\|\varphi\left(P_{\operatorname{ker}(X)}\right)-1\right\|<\varepsilon /(4\|M\|)$. With this $\delta$, (i) and (ii) are satisfied.

THEOREM 4.2. Suppose that $T$ has a local cross-section and that $S$ satisfies the following conditions: (i) $T^{*} T+S^{*} S$ is invertible, (ii) $S$ is right invertible, and (iii) $S$ is in $C^{*}(T)$. Then $M=M(T, S)$ has a local cross-section.

Proof. Let $(\psi, \mathscr{B}(T, \varepsilon))$ denote a cross-section for $T$. We use the notation and results of Lemma 4.1. Thus, if $X$ is in $\mathscr{B}(M, \delta)$ and $V=\varphi\left(P_{\text {ker }(X)}\right)$, then $\left\|V X V^{*}-M\right\|<\varepsilon$. Now $V^{*} P V=P_{\operatorname{ker}(X)}$, so $P_{\mathrm{ker}\left(V X V^{*}\right)}=V P_{\mathrm{ker}(X)} V^{*}=P$, and thus $V X V^{*}=W M W^{*}=M\left(T_{1}, S_{1}\right)$ where $W$ is unitary and $T_{1}^{*} T_{1}+$ $S_{1}^{*} S_{1}$ is invertible. It follows that $W P=P W$, and thus there exist unitary operators $Z$ and $Y$ such that $T_{1}=Z^{*} T Z$ and $S_{1}=Y^{*} S Z$. Since $\left\|T_{1}-T\right\|$ $<\left\|M\left(T_{1}, S_{1}\right)-M\right\|<\varepsilon$, we may now define 


$$
\rho(X)=\left(\psi\left(T_{1}\right) \oplus \psi\left(T_{1}\right) Z^{*} Y\right) \varphi\left(P_{\mathrm{ker}(X)}\right) .
$$

We will show that $(\rho, \mathscr{B}(M, \delta))$ is a local cross-section for $M$.

To check that $\rho$ is well defined, suppose there exist unitary operators $Z_{1}$ and $Y_{1}$ such that $Z_{1}^{*} T Z_{1}=Z^{*} T Z$ and $Y_{1}^{*} S Z_{1}=Y^{*} S Z$. Since $T$ commutes with $Z_{1} Z^{*}$, so does $S$, and thus $Y_{1}^{*} Z_{1} Z^{*} S Z=Y_{1}^{*} S Z_{1}=Y^{*} S Z=$ $Y^{*} Z Z^{*} S Z$. Now (ii) implies that $Y_{1}^{*} Z_{1}=Y^{*} Z$, and therefore $\rho$ is well defined. Since $\psi\left(T_{1}\right)^{*} T \psi\left(T_{1}\right)=T_{1}=Z^{*} T Z$, (iii) implies that $Z \psi\left(T_{1}\right)^{*}$ commutes with $S$. Now we have

$$
\begin{aligned}
\rho(X)^{*} M \rho(X) & =V^{*} M\left(\psi\left(T_{1}\right)^{*} T \psi\left(T_{1}\right), Y^{*} Z \psi\left(T_{1}\right)^{*} S \psi\left(T_{1}\right)\right) V \\
& =V^{*} M\left(T_{1}, S_{1}\right) V=X .
\end{aligned}
$$

Since $\rho(M)=1$, to complete the proof it suffices to verify that $\rho$ is continuous. Suppose that $X_{n} \rightarrow X_{0}$ in $\mathscr{B}(M, \delta)$. Lemma 4.1 implies that $\varphi\left(P_{\operatorname{ker}\left(X_{n}\right)}\right) \rightarrow$ $\varphi\left(P_{\text {ker }\left(X_{0}\right)}\right)$; moreover, if we set $V_{n}=\varphi\left(P_{\operatorname{ker}\left(X_{n}\right)}\right)$ for $n \geqslant 0$, then $V_{n} X_{n} V_{n}^{*} \rightarrow$ $V_{0} X_{0} V_{0}^{*}$, and each $V_{n} X_{n} V_{n}^{*}$ is of the form $M_{n}=M\left(T_{n}, S_{n}\right)$, where $T_{n}^{*} T_{n}+$ $S_{n}^{*} S_{n}$ is invertible. As above, there exist sequences $\left\{Z_{n}\right\},\left\{Y_{n}\right\} \subset \mathcal{U}(\mathcal{F C})$ such that $T_{n}=Z_{n}^{*} T Z_{n}$ and $S_{n}=Y_{n}^{*} S Z_{n}(n \geqslant 0)$. Since $T_{n} \rightarrow T_{0}$ in $\mathscr{B}(T, \varepsilon)$, then $\psi\left(T_{n}\right) \rightarrow \psi\left(T_{0}\right)$; it now suffices to prove that $Z_{n}^{*} Y_{n} \rightarrow Z_{0}^{*} Y_{0}$. From the remarks at the beginning of this section, (ii) and (iii) imply that there exists an operator $R$ in $C^{*}(T)$ such that $S R=1$, and since $Z_{n}^{*} T Z_{n} \rightarrow Z_{0}^{*} T Z_{0}$, it follows that $Z_{n}^{*} R Z_{n} \rightarrow Z_{0}^{*} R Z_{0}$. Since $Y_{n}^{*} Z_{n} Z_{n}^{*} S Z_{n}=S_{n} \rightarrow S_{0}=Y_{0}^{*} Z_{0} Z_{0}^{*} S Z_{0}$, it now follows that $Y_{n}^{*} Z_{n} \rightarrow Y_{0}^{*} Z_{0}$, which completes the proof.

THEOREM 4.3. If $T$ has a local cross-section and $S$ is an isometry in $\mathcal{L}(\mathcal{H C})$, then $M=M(T, S)$ has a local cross-section.

Proof. Let $(\psi, \mathscr{B}(T, \varepsilon))$ denote a local cross-section for $T$, where $0<\varepsilon<\frac{1}{2}$. We use the notation and results of Lemma 4.1. If $X$ is in $\mathscr{B}(M, \delta)$ and $V=\varphi\left(P_{\operatorname{ker}(X)}\right)$, then, as in the proof of the preceding result, $V X V^{*}=$ $M\left(T_{1}, S_{1}\right)$, where $T_{1}^{*} T_{1}+S_{1}^{*} S_{1}$ is invertible. It follows as before that there exist unitary operators $Z$ and $Y$ such that $T_{1}=Z^{*} T Z$ and $S_{1}=Y^{*} S Z$. Since $\left\|Z^{*} T Z-T\right\|<\varepsilon, \psi\left(T_{1}\right)$ is defined. We next define a unitary operator $W$ such that $W^{*} S=U \equiv Y^{*} S Z \psi\left(T_{1}\right)^{*}$. For each $t$ in $\mathcal{H}$ we set $W_{1}(S t)=U t$; $W_{1}$ is a linear mapping of $S \mathcal{H C}$ isometrically onto $U \mathcal{T C}$. Since $\left\|Y^{*} S Z-S\right\|$ $<\varepsilon$, then $\left\|P_{U \mathscr{C}}-P_{S \mathscr{C}}\right\|=\left\|U U^{*}-S S^{*}\right\|<2 \varepsilon<1$. Let $\left(\gamma, \mathfrak{B}\left(P_{S \mathscr{C}}, 1\right)\right)$ de-

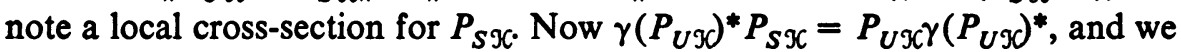
define $W_{2}:(S \mathcal{H C})^{\perp} \rightarrow(U \mathcal{H})^{\perp}$ by $W_{2}=\gamma\left(P_{U \mathscr{}}\right)^{*} \mid(S \mathcal{H C})^{\perp}$. Thus $W_{1}$ and $W_{2}$ together define a unitary operator $W_{3}$ on $\mathcal{K}$ such that if we set $W=W_{3}^{*}$, then $W^{*} S \psi\left(T_{1}\right)=Y^{*} S Z$. Furthermore, $W$ is independent of $Y$ and $Z$ since $W$ is defined in terms of $S_{1}=Y^{*} S Z$. We now define

$$
\tau(X)=\left(\psi\left(T_{1}\right) \oplus W\right) \varphi\left(P_{\mathrm{ker}(X)}\right),
$$


and we will show that $(\tau, \mathscr{B}(M, \delta))$ is a local cross-section. It is clear that $\pi_{M} \tau=1_{\mathscr{B}(M, \delta)}$ and that $\tau(M)=1$. Suppose that $X_{n} \rightarrow X_{0}$ in $\mathscr{B}(M, \delta)$. If $V_{n}=\varphi\left(P_{\text {ker }\left(X_{n}\right)}\right)(n \geqslant 0)$, then $V_{n} \rightarrow V_{0}, V_{n} X_{n} V_{n}^{*} \rightarrow V_{0} X_{0} V_{0}^{*}$, and each $V_{n} X_{n} V_{n}^{*}$ is of the form $M_{n}=M\left(Z_{n}^{*} T Z_{n}, Y_{n}^{*} S Z_{n}\right)$, where $Z_{n}$ and $Y_{n}$ are unitary. Since $Z_{n}^{*} T Z_{n} \rightarrow Z_{0}^{*} T Z_{0}$ in $\mathscr{B}(T, \varepsilon)$, then $\psi\left(Z_{n}^{*} T Z_{n}\right) \rightarrow \psi\left(Z_{0}^{*} T Z_{0}\right)$. Thus it suffices to prove that $W_{n} \rightarrow W_{0}$, where $W_{n}$ satisfies $W_{n}^{*} S=U_{n} \equiv$ $Y_{n}^{*} S Z_{n} \psi\left(Z_{n}^{*} T Z_{n}\right)^{*}(n \geqslant 0)$, and $W_{n}^{*}\left|(S \mathcal{H C})^{\perp}=\gamma\left(P_{U_{n}} \mathcal{S}\right)^{*}\right|(S \mathcal{H C})^{\perp}$. Let $P_{n}=$ $P_{U_{n}} s ;$ since $Y_{n}^{*} S Z_{n} \rightarrow Y_{0}^{*} S Z_{0}$, we have $\gamma\left(P_{n}\right) \rightarrow \gamma\left(P_{0}\right)$. For $\alpha>0$, let $N$ be a positive integer such that if $n>N$, then $\left\|U_{n}-U_{0}\right\|<\alpha$ and $\| \gamma\left(P_{n}\right)-$ $\gamma\left(P_{0}\right) \|<\alpha$. If $z$ is in $\mathcal{H},\|z\|=1$, then $z=S t+z^{\prime}$, where $z^{\prime}$ is in $(S \mathcal{H C})^{\perp}$ and $\|t\|^{2}+\left\|z^{\prime}\right\|^{2}=1$. Now

$$
\begin{aligned}
\left\|\left(W_{n}-W_{0}\right)^{*} z\right\|^{2} & =\left\|\left(U_{n}-U_{0}\right) t+\left(\gamma\left(P_{n}\right)^{*}-\gamma\left(P_{0}\right)^{*}\right) z^{\prime}\right\|^{2} \\
& <\alpha^{2}\left(\|t\|+\left\|z^{\prime}\right\|\right)^{2} \leqslant 2 \alpha^{2} .
\end{aligned}
$$

Thus $\left\|W_{n}-W_{0}\right\|<2^{1 / 2} \alpha$, and the proof is complete.

Straightforward modifications of the proofs of Theorem 4.2 and Theorem 4.3 yield the following results, whose proofs are omitted.

Proposition 4.4. If $T$ satisfies (P) and $S$ satisfies conditions (i)-(iii) of Theorem 4.2, then $M=M(T, S)$ satisfies (P).

Proposition 4.5. If $T$ satisfies (P) and $S$ is an isometry in $\mathcal{L}(\mathcal{F C})$, then $M=M(T, S)$ satisfies $(\mathrm{P})$.

REMARK. The preceding results, together with those of $\$ \S 2$ and 3, provide examples of nonnormal operators that satisfy $(\mathrm{P})$ or have local cross-sections. If we consider the classes of (1) algebraic operators, (2) operators with finite spectrum, (3) operators with a closed unitary orbit, (4) operators satisfying the sequential unitary lifting property, and (5) operators having a local cross-section, then our results, together with those of [6], show that for normal operators and isometries these five classes coincide. For arbitrary nonnormal operators, we have not been able to determine the relationships between all of these classes. In this regard, the following questions seem pertinent.

1. If an operator satisfies the sequential unitary lifting property, does it have a local cross-section; does it satisfy the unitary path lifting property?

2. If $T$ satisfies (P), is $U(T)$ closed? An affirmative answer to this question would of course render Proposition 2.2 vacuous; on the other hand, the answer is not obviously affirmative even for the operators given by the results of this section.

3. Does every operator that is unitarily equivalent to an $n \times n$ operator matrix, each of whose entries is a scalar multiple of $1_{\mathfrak{S O}}$ have a local cross-section? Theorem 4.2 implies an affirmative answer when $n=2$. We 
note also that not every operator with a local cross-section is unitarily equivalent to such a scalar valued operator matrix. If $U$ is a unilateral shift of multiplicity one in $\mathcal{L}(\mathcal{H})$ and $T=M(0, U)$, then Theorem 4.3 implies that $T$ has a local cross-section; moreover, $\operatorname{dim}\left(\operatorname{ker}(T) \cap \operatorname{ker}\left(T^{*}\right)\right)=1$. Now $T$ is not unitarily equivalent to a scalar valued operator matrix; indeed, if $S$ is unitarily equivalent to an $n \times n$ scalar valued operator matrix, then $\operatorname{dim}\left(\operatorname{ker}(S) \cap \operatorname{ker}\left(S^{*}\right)\right)=0$ or $\operatorname{dim}\left(\operatorname{ker}(S) \cap \operatorname{ker}\left(S^{*}\right)\right)=\aleph_{0} \quad$ (since $S$ is unitarily equivalent to $A \oplus A \oplus A \oplus \cdots \oplus A \oplus \cdots$, where $A$ is an operator on an $n$-dimensional Hilbert space).

4. Does each algebraic operator satisfy (P)? If Proposition 4.5 could be extended so that the isometry $S$ may be replaced by an arbitrary bounded operator acting between two possibly different Hilbert spaces, then we could prove that each algebraic operator satisfies (P).

ADDED IN PROOF. In a forthcoming sequel to this paper, question 2 is answered affirmatively. As a consequence, it is proved that each operator satisfying (P) is unitarily equivalent to an operator of the form $A \oplus B$ $\oplus \cdots \oplus B \oplus \cdots$, where $A$ and $B$ are operators on finite dimensional spaces. The conjecture on hyponormal operators following Corollary 3.7 is proved, and question 4 is answered negatively.

\section{REFERENCES}

1. H. O. Cordes, On a class of $C^{*}$-algebras, Math. Ann. 170 (1967), 283-313. MR 35 \#749.

2. L. A. Fialkow, A note on limits of unitarily equivalent partial isometries (preprint).

3. R. Gellar and L. Page, Limits of unitarily equivalent normal operators, Duke Math. J. 41 (1974), 319-322. MR 49 \#3581.

4. P. R. Halmos, Quasitriangular operators, Acta Sci. Math. (Szeged) 29 (1968), 283-293. MR 38 \#2627.

5. __ A Hilbert space problem book, Van Nostrand, Princeton, N.J., 1967. MR 34 \#8178.

6. __ Limits of shifts, Acta Sci. Math. (Szeged) 34 (1973), 131-139. MR 49 \#3576.

7. H. Radjavi and P. Rosenthal, Invariant subspaces, Springer-Verlag, New York, 1973.

8. N. Salinas, Reducing essential eigenvalues, Duke Math. J. 40 (1973), 561-580.

Department of Mathematics, Western Michigan University, Kalamazoo, Michigan 49008 